$$
\text { DOE/PC/95/Ss- T2 }
$$

TECHNICAL PROGRESS REPORT: January-March, 1996

\title{
Biochemical Removal of HAP Precursors from Coal \\ DE-AC22-95PC95155
}

Little Bear Laboratories, Inc.

Red Lodge, Montana

Executive Summary

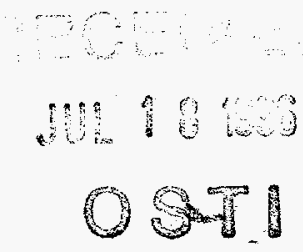

Shake flask bioleaching tests were conducted with Pittsburgh No. 8 and Indiana No. 5 coal. Bacteria removed pyritic sulfur from both coals at maximum rates of 5 to $9 \%$ per day, which was about 20 times the abiotic rate of pyrite oxidation. Concentrations of inorganic hazardous air pollutant (HAP) precursor elements in starting coal, bioleached coal and in leach solutions were measured. Of the 13 HAP precursors, significant amounts of arsenic, cobalt, cadmium, manganese, and nickel were removed from both coals by bacterial activity and also by the acidic leach solutions in control flasks. Little or no mercury, lead, beryllium, chromium, antimony, fluorine or chlorine was removed from the coals. Selenium was bioleached from both coals as determined by analysis of $\mathrm{Se}$ in leach solutions. However, analyses of $\mathrm{Se}$ in starting coal and in coal residues remains problematic. With very few exceptions, mass balances for the HAP precursors ranged from 80 to $120 \%$.

Concise Summary of Work Performed

Improved analytical methods were developed for measuring concentrations of $\mathrm{Hg}$, Se, $\mathrm{As}$, and $\mathrm{Sb}$ in coal. Shake flask tests with pyrite oxidizing bacteria were conducted on Pittsburgh No. 8 and Indiana No. 5 coal. Concentrations of HAP precursors in the starting coal, leach solutions, and final coal residues were measured. A column leaching-rotating biological contactor (RBC) unit was assembled and a column leach test with Pittsburgh No. 8 coal was begun.

Variances: No major variances to the work plan

Open Items: None

Forecast for Upcoming Quarter: Column-RBC tests with Pittsburgh No. 8 coal will be completed. Shake flask tests on two additional U.S. coals will be conducted. Methods for improved selenium measurement in coal will be investigated. A detailed plan will be finalized for testing HAP precursor removal in the slurry column reactor at the Idaho National Engineering Laboratory. A coal for these tests will be selected and arrangements made for its delivery to INEL.

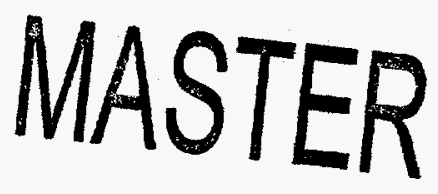




\section{DISCLAIMER}

Portions of this document may be illegible in electronic image products. Images are produced from the best available original document. 


\section{DISCLAIMER}

This report was prepared as an account of work sponsored by an agency of the United States Government. Neither the United States Government nor any agency thereof, nor any of their employees, makes any warranty, express or implied, or assumes any legal liability or responsibility for the accuracy, completeness, or usefulness of any information, apparatus, product, or process disclosed, or represents that its use would not infringe privately owned rights. Reference herein to any specific commercial product, process, or service by trade name, trademark, manufacturer, or otherwise does not necessarily constitute or imply its endorsement, recommendation, or favoring by the United States Government or any agency thereof. The views and opinions of authors expressed herein do not necessarily state or reflect those of the United States Government or any agency thereof. 
Technical and Scientific Results

1. Shake Flask Test Work-Pittsburgh No. 8 Coal

An ultimate and short proximate analysis was done on the starting coal (-100 mesh) along with a determination of forms of sulfur and concentration of 13 inorganic HAP precursors (Table 1). Duplicate flasks containing $20 \%$ slurries of Pittsburgh No. 8 coal were inoculated with pyrite oxidizing bacteria and incubated at $25^{\circ} \mathrm{C}$ with shaking at 180 r.p.m. until biooxidation of pyrite was nearly complete. Another set of duplicate flasks were uninoculated controls. An analysis was also done on the recovered product coals (Table 1). Selenium analyses of coals are incomplete (see discussion later in this report). Antimony and mercury analyses are in progress but were not completed as of March 31.

The kinetics of pyrite oxidation were estimated by the concentration of iron and sulfate in solution. The maximum rate of biological pyrite oxidation (Table 2) was determined from the slope of the $\mathrm{Fe}$ and sulfate dissolution curves (Figure 1).

Table 2. Maximum daily rate ${ }^{1}$ of pyrite oxidation (\%) with Pittsburgh No. 8 Coal

\begin{tabular}{|l|l|l|}
\hline & Based on solution $\mathrm{Fe}$ & Based on solution $\mathrm{SO}_{4}$ \\
\hline cells-1 & 8.4 & 8.0 \\
\hline cells-2 & 9.2 & 8.6 \\
\hline control-1 & 1.1 & 1.1 \\
\hline control-2 & 0.3 & 0.3 \\
\hline
\end{tabular}

${ }^{1}$ rate for cells based on data from day 4-12, rate for control based on all data (Fig.1)

Despite our attempts to sterilize the coal under argon at $100^{\circ} \mathrm{C}$, some growth of pyrite oxidizing bacteria began in control flasks during the time course. This growth was indicated by increasing $\mathrm{Fe}(\mathrm{III}) / \mathrm{Fe}$ (II) ratios and increases in the rate of iron and sulfate dissolution. At this point, $3 \mathrm{ml}$ of a bacteriocidal solution of $2 \%$ thymol in methanol was added to control flasks (day 12 in control 2 and day 15 in control 1). This treatment stopped bacterial pyrite oxidation. However, since some limited bacterial growth occurred in controls prior to biocide treatment, the rate of pyrite oxidation in control flasks, especially in control 1 , is somewhat higher than the strictly chemical rate of pyrite oxidation.

Over one third of the arsenic, cadmium, cobalt, manganese, and nickel were removed from Pittsburgh coal following biooxidation, based on analyses of the starting coal and biotreated and control coal residues (Table 1). With the exception of arsenic, over $20 \%$ of each of these elements was also removed from coal in control flasks. Chromium removal was 12 to $15 \%$ in biotreated coal and $4 \%$ in controls. 
Table 1. Analysis of Pittsburgh 8 Coal: Shake Flask Test (-100 mesh, dry basis)

\begin{tabular}{|c|c|c|c|c|c|}
\hline & Raw coal & Biotreated & Control & \%loss-microbial & $\%$ loss-control \\
\hline$\%$ carbon & 67.95 & $\begin{array}{l}70.35 \\
70.49\end{array}$ & $\begin{array}{l}69.43 \\
69.50\end{array}$ & & \\
\hline$\%$ hydrogen & 4.59 & $\begin{array}{l}4.53 \\
4.51\end{array}$ & $\begin{array}{l}4.52 \\
4.49\end{array}$ & & \\
\hline$\%$ nitrogen & 1.38 & $\begin{array}{l}1.37 \\
1.36\end{array}$ & $\begin{array}{l}1.33 \\
1.33\end{array}$ & & \\
\hline$\%$ sulfur & 2.69 & $\begin{array}{l}1.50 \\
1.53\end{array}$ & $\begin{array}{l}2.17 \\
2.29\end{array}$ & $\begin{array}{l}44 \\
43\end{array}$ & $\begin{array}{l}19 \\
15\end{array}$ \\
\hline$\%$ ash & 16.66 & $\begin{array}{l}14.82 \\
15.27\end{array}$ & $\begin{array}{l}16.03 \\
15.97\end{array}$ & $\begin{array}{r}11 \\
8\end{array}$ & $\begin{array}{l}4 \\
4\end{array}$ \\
\hline$\%$ oxygen (diff) & 6.73 & $\begin{array}{l}7.43 \\
6.84\end{array}$ & $\begin{array}{l}6.52 \\
6.42\end{array}$ & & \\
\hline Btu/lb & 12120 & $\begin{array}{l}12446 \\
12433\end{array}$ & $\begin{array}{l}12314 \\
12307\end{array}$ & & \\
\hline$\%$ pyritic sulfur & 1.17 & $\begin{array}{l}0.12 \\
0.12\end{array}$ & $\begin{array}{l}0.80 \\
0.87\end{array}$ & $\begin{array}{l}90 \\
90\end{array}$ & $\begin{array}{l}32 \\
26\end{array}$ \\
\hline$\%$ sulfate sulfur & 0.32 & $\begin{array}{l}0.24 \\
0.27 \\
\end{array}$ & $\begin{array}{l}0.22 \\
0.21\end{array}$ & & \\
\hline$\%$ org. S (diff) & 1.20 & $\begin{array}{l}1.14 \\
1.14 \\
\end{array}$ & $\begin{array}{l}1.15 \\
1.21 \\
\end{array}$ & & \\
\hline \multicolumn{6}{|l|}{ HAPS, ug/g coal } \\
\hline mercury & $\begin{array}{l}0.19 \\
0.16\end{array}$ & & & & \\
\hline arsenic & $\begin{array}{l}8.9 \\
8.4 \\
\end{array}$ & $\begin{array}{l}4.9 \\
4.4 \\
\end{array}$ & $\begin{array}{l}7.6 \\
8.8 \\
\end{array}$ & $\begin{array}{l}43 \\
49 \\
\end{array}$ & $\begin{array}{l}12 \\
0 \\
\end{array}$ \\
\hline cadmium & $\begin{array}{l}1.0 \\
1.1\end{array}$ & $\begin{array}{l}0.5 \\
0.6\end{array}$ & $\begin{array}{l}0.7 \\
0.7 \\
\end{array}$ & $\begin{array}{l}53 \\
46 \\
\end{array}$ & $\begin{array}{l}32 \\
30 \\
\end{array}$ \\
\hline chromium & $\begin{array}{l}29.1 \\
29.6 \\
\end{array}$ & $\begin{array}{l}25.1 \\
25.9 \\
\end{array}$ & $\begin{array}{l}28.1 \\
28.3 \\
\end{array}$ & $\begin{array}{l}15 \\
12 \\
\end{array}$ & $\begin{array}{l}4 \\
4 \\
\end{array}$ \\
\hline \multicolumn{6}{|l|}{ selenium } \\
\hline antimony & $\begin{array}{l}0.6 \\
0.5\end{array}$ & & & & \\
\hline beryllium & $\begin{array}{l}<0.2 \\
<0.2\end{array}$ & $\begin{array}{l}<0.2 \\
<0.2\end{array}$ & $\begin{array}{l}<0.2 \\
<0.2\end{array}$ & & \\
\hline cobalt & $\begin{array}{l}7.6 \\
7.3 \\
\end{array}$ & $\begin{array}{l}4.7 \\
4.7 \\
\end{array}$ & $\begin{array}{l}5.2 \\
5.3 \\
\end{array}$ & $\begin{array}{l}37 \\
37 \\
\end{array}$ & $\begin{array}{l}30 \\
29\end{array}$ \\
\hline lead & $\begin{array}{l}12.3 \\
14.3 \\
\end{array}$ & $\begin{array}{l}13.9 \\
13.2 \\
\end{array}$ & $\begin{array}{l}13.1 \\
12.3 \\
\end{array}$ & $\begin{array}{l}0 \\
1 \\
\end{array}$ & $\begin{array}{l}2 \\
8 \\
\end{array}$ \\
\hline manganese & $\begin{array}{l}63.5 \\
59.6 \\
\end{array}$ & $\begin{array}{l}22.6 \\
24.6\end{array}$ & $\begin{array}{l}29.0 \\
30.5 \\
\end{array}$ & $\begin{array}{l}63 \\
60 \\
\end{array}$ & $\begin{array}{l}53 \\
50 \\
\end{array}$ \\
\hline nickel & $\begin{array}{l}17.3 \\
17.4 \\
\end{array}$ & $\begin{array}{l}10.9 \\
11.3 \\
\end{array}$ & $\begin{array}{l}13.6 \\
13.6 \\
\end{array}$ & $\begin{array}{l}37 \\
35 \\
\end{array}$ & $\begin{array}{l}22 \\
22 \\
\end{array}$ \\
\hline chlorine (\%) & 0.08 & $\begin{array}{l}0.08 \\
0.08\end{array}$ & $\begin{array}{l}0.08 \\
0.07\end{array}$ & $\begin{array}{l}0 \\
0\end{array}$ & $\begin{array}{l}0 \\
12\end{array}$ \\
\hline fluorine & 120 & $\begin{array}{l}109 \\
133\end{array}$ & $\begin{array}{l}151 \\
138\end{array}$ & $\begin{array}{l}9 \\
0\end{array}$ & $\begin{array}{l}0 \\
0\end{array}$ \\
\hline
\end{tabular}

Duplicate values are for duplicate flasks, except for raw coal HAPs determinations which represent duplicate digestions. Some analyses on residues are not yet completed ( $\mathrm{Se}, \mathrm{Sb}, \mathrm{Hg}$ ). 
Figure 1. Kinetics of Pyrite Oxidation in Pittsburgh No. 8 Coal

Shake Flask Test, Pittsburgh No. 8 Coal $20 \%$ solids, -200 mesh
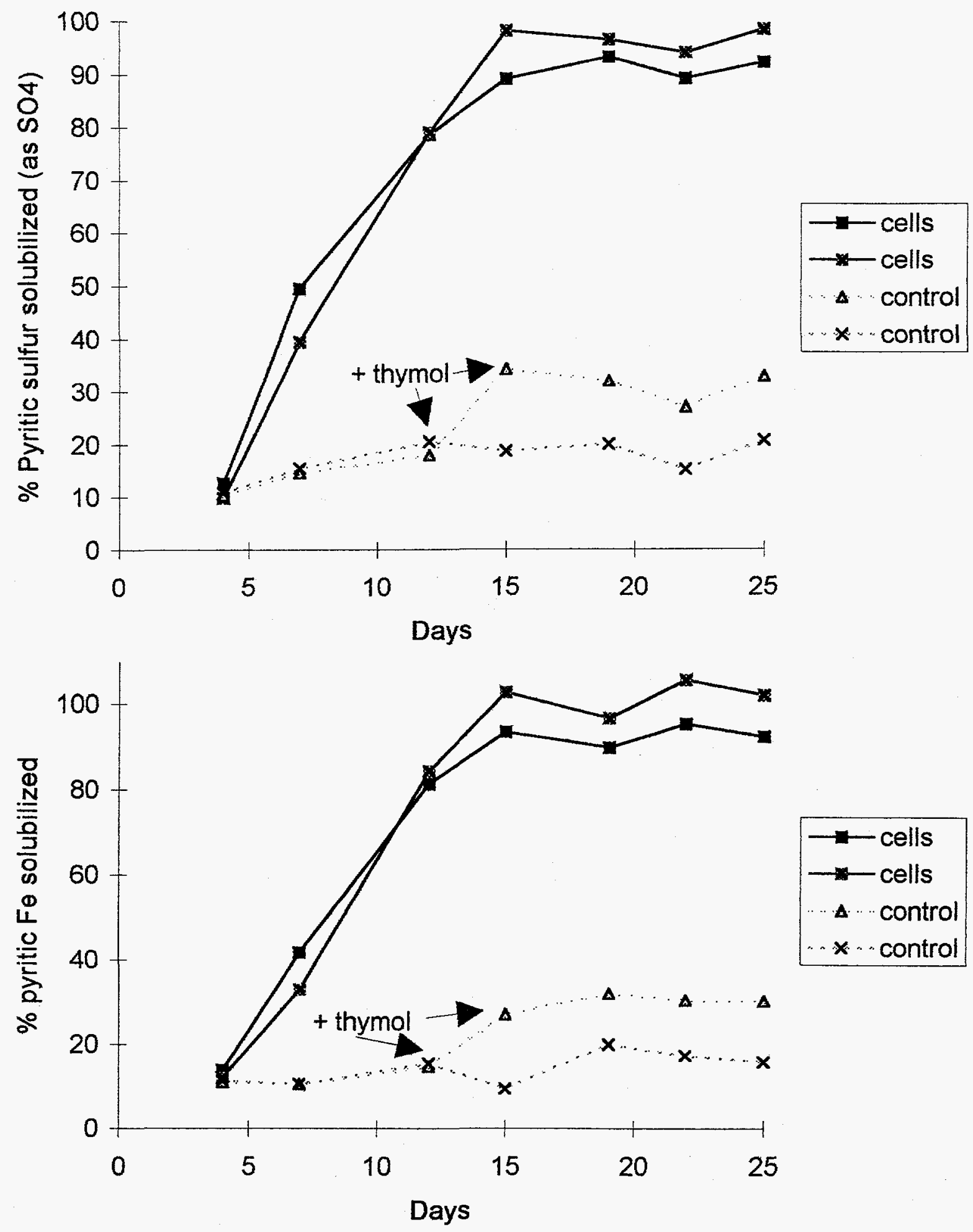
Less than $10 \%$ of fluorine, chlorine, and lead was removed from coal following biooxidation of the coal pyrite. Beryllium was not detected in the starting coal or in coal residues. Analyses of mercury, selenium and antimony in coal residues has yet to be completed.

HAP precursors were also measured in shake flask solutions at two time points during the test (after 11 and 15 days) and at the conclusion of the shake flask test. These solution measurements provided information for mass balances and for correlating pyrite oxidation with HAP precursor leaching. However, the two time point samples were taken after comparatively large amounts of pyrite had been biooxidized. Table 3 shows the extent of removal of HAP precursors in intermediate time point samples for the five elements which were significantly removed from coal in shake flask tests (As, $\mathrm{Cd}, \mathrm{Co}, \mathrm{Mn}, \mathrm{Ni}$ ). Note that most of the microbially catalyzed removal had occurred by the first time point sample when about $70 \%$ of the pyrite had been biooxidized. In some cases, the amount of metal in solubilized at the conclusion of the test was less than that shown for day 11 or day 15 . Possibly adsorption of metal to the coal occurred.

Table 3. \% of Coal HAP Precursors in Leach Solutions--Pittsburgh No. 8 Coal

\begin{tabular}{|l|l|l|l|}
\hline & Day 11 & Day 15 & Final \\
\hline \% pyrite biooxidation & 73 (cells) & 89 & 92 \\
(based on solution & 71 (cells) & 98 & 98 \\
sulfate concentration) & 17 (control) & 18 & 33 \\
& 19 (control) & 10 & 21 \\
\hline Arsenic & $60 \%$ & $62 \%$ & $47 \%$ \\
& 55 & 59 & 43 \\
& 1 & 16 & 11 \\
& 2 & 2 & 2 \\
\hline Cadmium & 31 & 30 & 31 \\
& 33 & 35 & 31 \\
& 17 & 20 & 19 \\
& 18 & 17 & 19 \\
\hline Cobalt & 35 & 33 & 28 \\
& 37 & 37 & 28 \\
& 30 & 29 & 25 \\
& 30 & 29 & 24 \\
\hline Manganese & 57 & 61 & 47 \\
& 61 & 57 & 47 \\
& 45 & 46 & 41 \\
& 44 & 45 & 40 \\
\hline Nickel & 35 & 33 & 29 \\
& 38 & 37 & 30 \\
& 26 & 26 & 23 \\
& 26 & 26 & 22 \\
\hline
\end{tabular}

Values in above boxes are for duplicate inoculated (first two values) and control (second two values) flasks. Values are $\%$ of HAP precursor in coal that has been solubilized. 
Mass balances were calculated for each HAP precursor (Table 4). The total mass of each HAP precursor was determined by multiplying its concentration in starting coal by the weight of starting coal. This mass was compared to the amount recovered in residue plus leach solutions. Mass balances were 82 to $113 \%$, with the exception of cadmium in one biotreated flask (75\%), and fluorine in 3 of the 4 flasks $(127-138 \%)$. The \% loss of metal (by solution) generally agreed well with the \% loss of metal determined by starting coal and residue coal analysis.

It was surprising that a greater extent of metal removal from coal was not achieved, since many of the HAP precursors are thought to be associated primarily with pyrite or as sulfides, and given that $90 \%$ of the pyrite was removed from the coal. Perhaps some readsorption of solubilized metal occurred (see discussion above related to table 3 ). It is worthwhile to determine whether coals can adsorb HAP precursors from solution. A experiment will be conducted in the coming quarter with Pittsburgh coal and acidic bioleach solutions to test this hypothesis. Should significant adsorption occur, it will be worthwhile to consider process steps to minimize adsorption or to strip metals with an improved washing step. 
Table 4. Mass Balances for Pittsburgh 8 Shake Flask Test*

\begin{tabular}{|c|c|c|c|c|c|}
\hline & $\begin{array}{l}\text { starting coal } \\
\mathrm{mg}\end{array}$ & $\begin{array}{l}\text { solution } \\
\mathrm{mg}\end{array}$ & $\begin{array}{l}\text { final coal } \\
\text { mg }\end{array}$ & $\%$ recovery & $\begin{array}{l}\% \text { loss (by } \\
\text { solution) }\end{array}$ \\
\hline $\begin{array}{ll}\mathrm{Hg} & \text { cells } \\
& \text { cells } \\
& \text { control } \\
& \text { control } \\
\end{array}$ & $\begin{array}{l}0.0060 \\
0.0060 \\
0.0060 \\
0.0060 \\
\end{array}$ & $\begin{array}{l}<0.0001 \\
<0.0001 \\
<0.0001 \\
<0.0001\end{array}$ & & & \\
\hline arsenic & $\begin{array}{l}0.259 \\
0.260 \\
0.259 \\
0.259\end{array}$ & $\begin{array}{l}0.120 \\
0.111 \\
0.028 \\
0.004\end{array}$ & $\begin{array}{l}0.143 \\
0.127 \\
0.225 \\
0.262\end{array}$ & $\begin{array}{r}102 \\
92 \\
98 \\
103\end{array}$ & $\begin{array}{r}46 \\
43 \\
11 \\
2\end{array}$ \\
\hline cadmium & $\begin{array}{l}0.032 \\
0.032 \\
0.032 \\
0.032 \\
\end{array}$ & $\begin{array}{l}0.010 \\
0.010 \\
0.006 \\
0.006\end{array}$ & $\begin{array}{l}0.014 \\
0.017 \\
0.021 \\
0.022 \\
\end{array}$ & $\begin{array}{l}75 \\
84 \\
84 \\
88 \\
\end{array}$ & $\begin{array}{l}31 \\
31 \\
19 \\
19 \\
\end{array}$ \\
\hline chromium & $\begin{array}{l}0.882 \\
0.884 \\
0.882 \\
0.882 \\
\end{array}$ & $\begin{array}{l}0.035 \\
0.034 \\
0.019 \\
0.015 \\
\end{array}$ & $\begin{array}{l}0.729 \\
0.754 \\
0.833 \\
0.846 \\
\end{array}$ & $\begin{array}{l}87 \\
89 \\
97 \\
98 \\
\end{array}$ & $\begin{array}{l}4 \\
4 \\
2 \\
2 \\
\end{array}$ \\
\hline selenium & & $\begin{array}{l}0.005 \\
0.005 \\
<0.001 \\
<0.001\end{array}$ & & & \\
\hline antimony & $\begin{array}{l}0.017 \\
0.017 \\
0.017 \\
0.017 \\
\end{array}$ & $\begin{array}{l}<0.001 \\
<0.001 \\
<0.001 \\
<0.001\end{array}$ & & & \\
\hline beryllium & $\begin{array}{l}<0.006 \\
<0.006 \\
<0.006 \\
<0.006\end{array}$ & $\begin{array}{l}0.002 \\
0.002 \\
0.002 \\
0.002 \\
\end{array}$ & $\begin{array}{l}<0.006 \\
<0.006 \\
<0.006 \\
<0.006 \\
\end{array}$ & & \\
\hline cobalt & $\begin{array}{l}0.225 \\
0.225 \\
0.225 \\
0.225 \\
\end{array}$ & $\begin{array}{l}0.063 \\
0.064 \\
0.056 \\
0.055 \\
\end{array}$ & $\begin{array}{l}0.135 \\
0.136 \\
0.153 \\
0.159\end{array}$ & $\begin{array}{l}88 \\
89 \\
93 \\
95\end{array}$ & $\begin{array}{l}28 \\
28 \\
25 \\
24\end{array}$ \\
\hline lead & $\begin{array}{l}0.399 \\
0.399 \\
0.399 \\
0.399 \\
\end{array}$ & $\begin{array}{l}0.025 \\
0.025 \\
0.016 \\
0.013 \\
\end{array}$ & $\begin{array}{l}0.404 \\
0.384 \\
0.388 \\
0.367 \\
\end{array}$ & $\begin{array}{l}108 \\
103 \\
101 \\
95 \\
\end{array}$ & $\begin{array}{l}6 \\
6 \\
4 \\
3 \\
\end{array}$ \\
\hline manganese & $\begin{array}{l}1.848 \\
1.853 \\
1.848 \\
1.848 \\
\end{array}$ & $\begin{array}{l}0.863 \\
0.874 \\
0.761 \\
0.737 \\
\end{array}$ & $\begin{array}{l}0.657 \\
0.715 \\
0.859 \\
0.911 \\
\end{array}$ & $\begin{array}{l}82 \\
86 \\
88 \\
89 \\
\end{array}$ & $\begin{array}{l}47 \\
47 \\
41 \\
40 \\
\end{array}$ \\
\hline nickel & $\begin{array}{l}0.523 \\
0.524 \\
0.523 \\
0.523\end{array}$ & $\begin{array}{l}0.153 \\
0.156 \\
0.120 \\
0.117\end{array}$ & $\begin{array}{l}0.317 \\
0.328 \\
0.403 \\
0.406\end{array}$ & $\begin{array}{l}90 \\
92 \\
100 \\
100\end{array}$ & $\begin{array}{l}29 \\
30 \\
23 \\
22\end{array}$ \\
\hline chlorine & $\begin{array}{l}24 \\
24 \\
24 \\
24 \\
\end{array}$ & $\begin{array}{l}2 \\
4 \\
0 \\
3 \\
\end{array}$ & $\begin{array}{l}23 \\
23 \\
24 \\
21 \\
\end{array}$ & $\begin{array}{l}104 \\
113 \\
100 \\
113 \\
\end{array}$ & $\begin{array}{l}8 \\
17 \\
0 \\
13 \\
\end{array}$ \\
\hline fluorine & $\begin{array}{l}3.60 \\
3.60 \\
3.60 \\
3.60 \\
\end{array}$ & $\begin{array}{l}0.83 \\
0.77 \\
0.51 \\
0.45 \\
\end{array}$ & $\begin{array}{l}3.17 \\
3.87 \\
4.47 \\
4.12 \\
\end{array}$ & $\begin{array}{l}111 \\
129 \\
138 \\
127 \\
\end{array}$ & $\begin{array}{l}23 \\
21 \\
14 \\
13 \\
\end{array}$ \\
\hline
\end{tabular}

*Four values are shown for each HAP precursor. As shown for $\mathrm{Hg}$ above, the first two rows are for the two inoculated flasks, the last two rows are for control flasks 
2. Shake Flask Test Work--Indiana No. 5 Coal

As with Pittsburgh coal, an ultimate analysis was done on the starting coal (-100 mesh) along with a determination of forms of sulfur and concentration of HAP precursors (Table 5). Some analyses were not yet available at the end of the quarter (empty boxes below).

Table 5. Analysis of Indiana 5 coal: Shake Flask Test (-100 mesh, dry basis)

\begin{tabular}{|c|c|c|c|c|c|}
\hline & Raw coal & Biotreated & Control & \%loss-microbial & $\%$ loss-control \\
\hline$\%$ carbon & 70.67 & & & & \\
\hline$\%$ hydrogen & 4.21 & & & & \\
\hline$\%$ nitrogen & 1.44 & & & & \\
\hline$\%$ sulfur & 4.09 & & & & \\
\hline$\%$ ash & 12.4 & & & & \\
\hline $\begin{array}{l}\% \text { oxygen } \\
\text { (difference) }\end{array}$ & 7.19 & & & & \\
\hline Btu/lb & 12519 & & & & \\
\hline$\%$ pyritic sulfur & 2.21 & & & & \\
\hline$\%$ sulfate sulfur & 0.14 & & & & \\
\hline $\begin{array}{l}\% \text { organic S } \\
\text { (difference) }\end{array}$ & 1.74 & & & & \\
\hline \multicolumn{6}{|l|}{ HAPS, ug/g coal } \\
\hline mercury & $\begin{array}{l}0.11 \\
0.12 \\
\end{array}$ & $\begin{array}{l}0.13 \\
0.11 \\
\end{array}$ & $\begin{array}{l}0.11 \\
0.13\end{array}$ & $\begin{array}{l}0 \\
0\end{array}$ & $\begin{array}{l}0 \\
0\end{array}$ \\
\hline arsenic & $\begin{array}{l}5.5 \\
5.7\end{array}$ & & & & \\
\hline cadmium & $\begin{array}{l}1.2 \\
1.2 \\
\end{array}$ & $\begin{array}{l}0.4 \\
0.7\end{array}$ & $\begin{array}{l}0.5 \\
0.8\end{array}$ & $\begin{array}{l}66 \\
45\end{array}$ & $\begin{array}{l}59 \\
32\end{array}$ \\
\hline chromium & $\begin{array}{l}12.2 \\
11.6 \\
\end{array}$ & $\begin{array}{l}11.9 \\
12.8 \\
\end{array}$ & $\begin{array}{l}12.6 \\
12.8 \\
\end{array}$ & $\begin{array}{l}0 \\
0 \\
\end{array}$ & $\begin{array}{l}0 \\
0 \\
\end{array}$ \\
\hline \multicolumn{6}{|l|}{ selenium } \\
\hline antimony & $\begin{array}{l}0.6 \\
0.7\end{array}$ & & & & \\
\hline beryllium & $\begin{array}{l}0.8 \\
0.8\end{array}$ & $\begin{array}{l}0.8 \\
0.7\end{array}$ & $\begin{array}{l}0.9 \\
0.7\end{array}$ & $\begin{array}{l}6 \\
12\end{array}$ & $\begin{array}{l}0 \\
17\end{array}$ \\
\hline cobalt & $\begin{array}{l}4.8 \\
4.4 \\
\end{array}$ & $\begin{array}{l}2.6 \\
3.4 \\
\end{array}$ & $\begin{array}{l}3.3 \\
2.6 \\
\end{array}$ & $\begin{array}{l}44 \\
27 \\
\end{array}$ & $\begin{array}{l}28 \\
44 \\
\end{array}$ \\
\hline lead & $\begin{array}{l}9.7 \\
9.4 \\
\end{array}$ & $\begin{array}{l}9.1 \\
9.2 \\
\end{array}$ & $\begin{array}{l}10.7 \\
9.6 \\
\end{array}$ & $\begin{array}{l}6 \\
2 \\
\end{array}$ & $\begin{array}{l}0 \\
0 \\
\end{array}$ \\
\hline manganese & $\begin{array}{l}39.1 \\
43.1\end{array}$ & $\begin{array}{l}14.8 \\
21.0\end{array}$ & $\begin{array}{l}17.3 \\
19.0\end{array}$ & $\begin{array}{l}64 \\
49\end{array}$ & $\begin{array}{l}58 \\
54\end{array}$ \\
\hline nickel & $\begin{array}{l}16.0 \\
15.6 \\
\end{array}$ & $\begin{array}{l}10.8 \\
14.7\end{array}$ & $\begin{array}{l}15.6 \\
10.9 \\
\end{array}$ & $\begin{array}{l}32 \\
7 \\
\end{array}$ & $\begin{array}{l}1 \\
31 \\
\end{array}$ \\
\hline chlorine (\%) & 0.01 & & & & \\
\hline fluorine & 70 & & & & \\
\hline
\end{tabular}


Duplicate inoculated and uninoculated flasks containing $20 \%$ slurries ( $40 \mathrm{~g}$ coal, $160 \mathrm{ml}$ of modified Kelly medium) of minus 100 mesh Indiana No. 5 coal were incubated at $25^{\circ} \mathrm{C}$ with shaking at 180 r.p.m. until biooxidation of pyrite was nearly complete. Uninoculated flasks were treated with $3 \mathrm{ml}$ of $2 \%$ thymol in methanol at the beginning of the test. An analysis was also done on the recovered product coals (Table 5).

The kinetics of pyrite oxidation were somewhat slower than with Pittsburgh coal and are shown Table 6 and Figure 2.

Table 6. Maximum daily rate ${ }^{1}$ of pyrite oxidation (\%) with Indiana No. 5 Coal

\begin{tabular}{|l|l|l|}
\hline & Based on solution $\mathrm{Fe}$ & Based on solution $\mathrm{SO}_{4}{ }_{4}$ \\
\hline cells-1 & 6.1 & 5.0 \\
\hline cells-2 & 5.2 & 4.6 \\
\hline control-1 & 0.3 & 0.5 \\
\hline control-2 & 0.5 & 0.3 \\
\hline
\end{tabular}

${ }^{1}$ rate for cells based on data from day 6-18 (day 15 omitted), rate for control based on all data (see Fig. 2)

As with Pittsburgh coal, significant amounts of cadmium, cobalt, manganese and nickel were removed in biotreated coal (although the agreement of duplicates was poor with respect to nickel content of bioprocessed and control coal). To a lesser extent these elements were also leached from coal in control flasks. Little or no mercury, chromium, beryllium or lead was removed from coal. Coal residue analyses for $\mathrm{F}, \mathrm{Cl}, \mathrm{As}$, Se and $\mathrm{Sb}$ were not yet completed, although solution arsenic measurements indicate biooxidation significantly increased arsenic removal compared to controls.

Time point samples were taken for Indiana No. 5 coal as they were for Pittsburgh No. 8 coal. Results for the five HAP precursors that were significantly leached are shown in Table 7. Again, the time points were taken after considerable pyrite oxidation occurred. Most of the HAPs were leached at the first time point. The first flask shown on day 18 has an anomalously high $\mathrm{Mn}$ value. The data were reexamined, but no explanation is obvious for the elevated value.

Mass balances (Table 8 ) were generally between $80 \%$ and $120 \%$. Two cadmium flasks had low values $(60 \%, 63 \%)$. 
Figure 2. Kinetics of Pyrite Biooxidation in Indiana No. 5 Coal
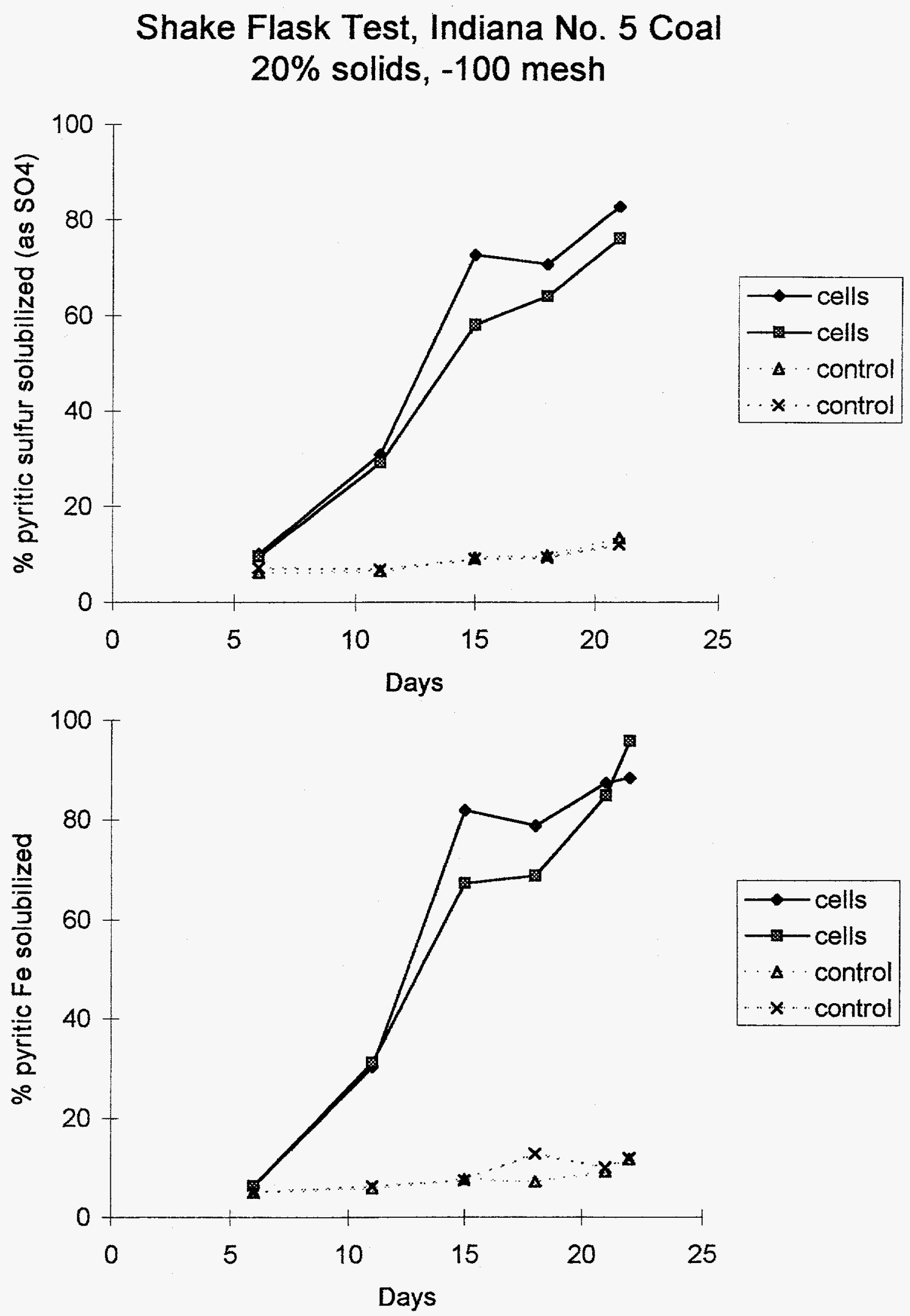
Table 7. \% of Coal HAP Precursors in Leach Solutions--Indiana No. 5 Coal

\begin{tabular}{|l|l|l|l|}
\hline & Day 16 & Day 18 & Final \\
\hline \% pyrite biooxidation & 72 (cells) & 79 & 83 \\
(based on solution & 61 (cells) & 69 & 76 \\
sulfate concentration) & 9 (control) & 10 & 13 \\
& 9 (control) & 9 & 12 \\
\hline Arsenic & $34 \%$ & $37 \%$ & $44 \%$ \\
& 29 & 37 & 41 \\
& 1 & 1 & 1 \\
& 1 & 1 & 1 \\
\hline Cadmium & 28 & 26 & 31 \\
& 28 & 26 & 34 \\
& 19 & 18 & 22 \\
& 16 & 18 & 23 \\
\hline Cobalt & 41 & 42 & 46 \\
& 41 & 39 & 45 \\
& 32 & 31 & 33 \\
& 30 & 30 & 32 \\
\hline Manganese & 55 & 97 & 62 \\
& 54 & 53 & 60 \\
& 42 & 43 & 42 \\
& 45 & 44 & 69 \\
\hline Nickel & 32 & 33 & 39 \\
& 31 & 31 & 38 \\
& 16 & 16 & 17 \\
\hline
\end{tabular}

Values in above boxes are for duplicate inoculated (first two values) and control (second two values) flasks. Values are \% of HAP precursor in coal that has been solubilized 
Table 8. Mass Balances--Indiana 5 Shake Flask Test

\begin{tabular}{|l|l|l|l|l|l|}
\hline & $\begin{array}{l}\text { starting coal } \\
\mathrm{mg}\end{array}$ & $\begin{array}{l}\text { solution } \\
\mathrm{mg}\end{array}$ & $\begin{array}{l}\text { final coal } \\
\mathrm{mg}\end{array}$ & $\begin{array}{l}\text { \% recovery } \\
\text { \% loss (by } \\
\text { solution) }\end{array}$ \\
\hline mercury & 0.0048 & $<0.0002$ & 0.0047 & 98 & $<4$ \\
& 0.0048 & $<0.0002$ & 0.0041 & 85 & $<4$ \\
& 0.0048 & $<0.0002$ & 0.0049 & 102 & $<4$ \\
& 0.0048 & $<0.0002$ & 0.0054 & 113 & $<4$ \\
\hline arsenic & 0.224 & & & & \\
\hline cadmium & 0.048 & 0.015 & 0.015 & 63 & 31 \\
& 0.048 & 0.016 & 0.025 & 85 & 33 \\
& 0.048 & 0.011 & 0.018 & 60 & 23 \\
& 0.048 & 0.011 & 0.030 & 85 & 23 \\
\hline chromium & 0.476 & 0.021 & 0.499 & 109 & 4 \\
& 0.476 & 0.020 & 0.487 & 107 & 4 \\
& 0.476 & 0.012 & 0.472 & 102 & 3 \\
& 0.476 & 0.018 & 0.464 & 101 & 4 \\
\hline selenium & & & & & \\
\hline antimony & 0.028 & & & & \\
\hline beryllium & 0.033 & 0.003 & 0.028 & 94 & 9 \\
& 0.033 & 0.004 & 0.027 & 94 & 12 \\
& 0.033 & 0.003 & 0.033 & 109 & 9 \\
& 0.033 & 0.003 & 0.025 & 85 & 9 \\
\hline cobalt & 0.190 & 0.087 & 0.093 & 95 & 46 \\
& 0.190 & 0.085 & 0.125 & 111 & 45 \\
& 0.190 & 0.063 & 0.123 & 98 & 33 \\
& 0.190 & 0.060 & 0.093 & 81 & 32 \\
\hline lead & 0.384 & 0.038 & 0.330 & 96 & 10 \\
& 0.384 & 0.038 & 0.345 & 100 & 10 \\
& 0.384 & 00.005 & 0.400 & 104 & $<1$ \\
& 0.384 & $<0.005$ & 0.347 & 90 & $<1$ \\
\hline manganese & 1.645 & 1.021 & 0.536 & 95 & 62 \\
& 1.645 & 0.981 & 0.786 & 107 & 60 \\
& 1.645 & 0.688 & 0.648 & 81 & 42 \\
& 1.645 & 1.138 & 0.689 & 111 & 69 \\
\hline nickel & 0.633 & 0.246 & 0.393 & 101 & 39 \\
& 0.633 & 0.241 & 0.550 & 125 & 38 \\
& 0.633 & 0.110 & 0.583 & 109 & 17 \\
\hline chlorine & 0.632 & 0.108 & 0.395 & 80 & 17 \\
\hline fluorine & 4.80 & & & & \\
\hline & & & & & \\
\hline
\end{tabular}

\section{Analytical Developments}

Although analysis of HAP precursors in leach solutions is straightforward, digestions are required to prepare solid samples (raw coal and recovered coal residues) for analysis of most of the HAP precursors. As discussed in the first quarter report, a digestion based on a modification of ASTM D3683 works well for most of the HAP precursors. However, that report indicated some issues were not resolved in the 
analyses of 3 of the HAP precursors: $\mathrm{Hg}$, Se and $\mathrm{Sb}$. As described below, these issues are largely resolved: EPA method 7471 is now being used for mercury analysis and Eschka oxidation for $\mathrm{As}$ and $\mathrm{Sb}$ analysis. Se remains problematic.

Mercury. Initially we used a refluxing sulfuric acid nitric acid method (U.S. Bureau of Mines RI 7609) to digest coal prior to analysis of mercury by cold vapor atomic absorption spectrophotometry (CVAAS). We have since adopted a more rapid digestion method, EPA 7471, which involves heating in aqua regia followed by permanganate treatment.

According to results of an interlaboratory study of the analysis of mercury in coal conducted by CONSOL, comparable results were obtained by method 7471 , oxygen bomb combustion (ASTM D3684), neutron activation analysis and atomic fluorescence analysis (reported in Energeia, vol 6, No. 5, 1995).

We found comparable $\mathrm{Hg}$ results in our analysis of starting Indiana 5 coal using either EPA method 7471 or the more vigorous Bureau of Mines digestion procedure. However, our mercury results were less than one tenth of the expected value for Indiana 5 coal. According to information supplied with the Indiana 5 coal by the Illinois Basin Coal Sample Program, the Indiana 5 coal sample (IBC-110) contains nearly 2 $\mathrm{ug} / \mathrm{g}$ of $\mathrm{Hg}$. Given this result and the lack of a coal standard certified for mercury content, we sent some coal samples to Huffman Laboratories for comparative analyses. Ron Kyle, Inorganic Lab Director at Huffman, indicates they use a nitric acid-refluxing perchloric acid digestion followed by CVAAS. As shown in Table 9 below, our results are close to those determined by Huffman. To date we have analyzed Pittsburgh No. 8 coal for its mercury content using only the Bureau of Mines digestion followed by CVAAS.

Table 9. Mercury (ug/g) in Duplicate Coal Samples Analyzed at Huffman Labs and at Little Bear Laboratories

\begin{tabular}{|l|l|ll|}
\hline coal sample & Huffman & LBL $^{1}$ & (s.d.) \\
\hline \hline Pitt 8 starting coal & 0.16 & 0.18 & $(0.02)$ \\
\hline Ind 5 starting coal & 0.12 & 0.11 & $(0.00)$ \\
\hline Ind 5 biooxidized residue & 0.13 & 0.13 & $(0.01)$ \\
\hline Ind 5 dup biooxidized residue & 0.11 & 0.11 & $(0.01)$ \\
\hline Ind 5 control residue & 0.13 & 0.11 & $(0.01)$ \\
\hline Ind 5 dup control residue & 0.15 & 0.13 & $(0.01)$ \\
\hline
\end{tabular}

${ }^{1}$ LBL method is EPA 7471 (Ind 5) or Bureau of Mines RI 7609 (Pitt 8)

${ }^{2}$ standard deviation for triplicate digestions

Antimony. This element is present in NIST SRM $1632 \mathrm{~b}$ at our detection limit $(0.2 \mathrm{ug} / \mathrm{g})$. Consequently, we relied on spike recovery of antimony to assess the analytical method. As reported in the first quarter report, post digestion spikes of antimony into the matrix 
resulting from D3683 digestion were poorly recovered. Modification of hydridization conditions failed to improve Sb recovery, so we turned our attention to an Eschka procedure. Despite initial poor spike recoveries, the matrix resulting from Eschka ignition gave good $\mathrm{Sb}$ recovery. Finally, we modified the Eschka oxidation procedure, placing crucibles into a hot muffle furnace. This resulted in $>80 \%$ spike recoveries of antimony. Furthermore, arsenic determined under these conditions in NIST SRM $1632 \mathrm{~b}$ was close to $100 \%$. Consequently, the hot muffle furnace Eschka oxidation will be used for the analysis of $\mathrm{As}$ and $\mathrm{Sb}$ in coal.

Selenium. The modified Eschka analysis above made only slight improvements in selenium spike recovery. Though post Eschka spikes of Se into the analysis solution are recovered at near $100 \%$, pre Eschka spikes of Se are recovered at only 10 to $20 \%$. $\mathrm{Se}$ is apparently volatilized during Eschka treatment. It may be possible to quantify Se in acid digests of coal. Se determinations in aqua regia digests of NIST coal will be tested next.

\section{Assembly of Bench Scale Leach Column-Rotating Biological Contactor (RBC)}

By the end of the quarter a system had been assembled to conduct bench scale column leaching tests. A test with $28 \times 100$ mesh Pittsburgh coal was begun at the end of the quarter. The details of the experiment will be described in the next report.

The column-RBC system consists of pairs of plastic columns of $300 \mathrm{ml}$ capacity, $4 \times 30$ $\mathrm{cm}$ in size. The columns are positioned above plastic reservoirs. Leach solution is percolated through the columns through the use of a multichannel peristaltic pump. A gear motor turns a series of $7 \mathrm{~cm}$ diameter plastic disks at $36 \mathrm{rpm}$. The disks are partially submerged in the reservoirs. Biofilms of iron oxidizing bacteria should develop on the disks, and the RBC should maintain sufficient aeration of the system to permit efficient iron biooxidation. The ferric-rich solution will then be pumped to the top of the columns.

\section{Effects of Results on Future Work}

The project is proceeding on schedule. HAP precursor quantitation in coal is, for the most part, proceeding well with good mass balances. Only selenium continues to present analytical difficulties. Additional work will be devoted to this problem in April. Samples were taken too late in the Indiana No. 5 and Pittsburgh No. 8 shake flask tests to determine the extent of HAP precursor leaching in the early stages of biodepyritization. Better timing of sampling of solutions for HAP precursor analysis will be required in future shake flask and leach column tests.

A determination of binding of HAP precursors in solution to coal will be made. This finding will be important in determining the effectiveness of bioleaching of these elements from coal and for process design and operation. 